\title{
Déterminisme génétique de la croissance en hauteur du Pin maritime (Pinus pinaster Ait.)
}

\section{I. - Rôle du polycyclisme}

\author{
A. KREMER \\ Laboratoire d'Amélioration des Arbres forestiers, I.N.R.A., \\ Pierroton, 33610 Cestas
}

\begin{abstract}
Résumé
A partir de l'information issue de plusieurs plantations expérimentales (test clonal, test de descendances maternelles et biparentales), et d'observations pluriannuelles et multistationnelles l'auteur étudie le polycyclisme en le décomposant en deux caractères :

- fréquence des seconds cycles (F);

- ćlongation de la seconde pousse quand elle s'exprime (E).

Chacun d'entre eux est sous contrôle génétique, stable dans le temps et dans l'espace. Cependant la stabilité est meilleure pour $\mathbf{F}$ que pour $\mathbf{E}$.

L'impact du polycyclisme sur la croissance annuelle est important : les polycycliques ont un avantage très net sur les monocycliques. L'avantage est cumulé sur plusieurs années si l'expression d'un second cycle est conservée. Il augmente également avec une amélioration du milieu.

Les corrélations phénotypiques entre accroissements primaire et secondairc sont négatives ou positives mais le plus généralement nulles quelles que soient les années incriminées. Pour une même année, au plan génétique, les valeurs sont tout aussi discordantes.

L'ensemble de ces résultats traduit l'intervention de mécanismes très différents dans l'expression du polycyclisme (facteurs du milieu, expressions morphogénétiques, modalités de croissance). Enfin, le polycyclisme crée un bruit de fond dans l'estimation des paramètres génétiques (diminution des héritabilités et augmentation de la variance de dominance).
\end{abstract}

Mots-ClÉs : Polycyclisme, croissance en hauteur, Pinus pinaster, contrôle génétiqué, stabilité.

\section{1. - Introduction}

Le choix d'un critère de sélection dans un programme d'amélioration est déterminé par l'importance économique et le déterminisme génétique du caractère en cause.

En ce qui concerne les espèces pérennes, ce choix passe par la recherche du meilleur critère prédicteur du caractère impliqué. Une compréhension précise du déter- 
minisme physiologique du caractère final est un atout majeur dans une telle démarche. Ce cas est cependant rare chez les arbres forestiers. L'alternative est alors d'utiliser dans un contexte bien défini, le critère jouissant du plus fort pouvoir de prédiction; la notion de prédiction est ici tout à fait générale, elle inclut bien évidemment la relation liant les deux caractères dans des conditions bien définies mais aussi la fiabilité d'un même critère d'un milieu à un autre. Par exemple, dans le cadre d'une sélection pour les valeurs génétiques additives, l'outil de la génétique quantitative permet de rechercher les critères (corrélations génétiques, coefficient de prédiction génétique), sans pour autant préjuger de la relation physiologique ou même génétique liant les deux caractères. Le critère de sélection devient ainsi une notion toute relative.

Chez le Pin maritime l'exemple de la production en bois est éloquent à ce sujet. Le prédicteur choisi pour sélectionner l'aptitude à la croissance en volume est l'accroissement en hauteur (très corrélé génétiquement au volume, plus héritable que l'accroissement en diamètre, et peu influencé par la concurrence). Cependant les premiers tests de descendances ont montré que les accroissements en hauteur jusqu'à la cinquième année étaient fortement entachés d'effets maternels (grosseur de graines) et masquaient les effets génétiques (BARADAT, 1975). Le choix s'est donc porté sur le cumul d'un maximum d'accroissements annuels au-delà de la cinquième année.

Il est fort probable que dans l'avenir ce choix évoluera encore (utilisation de la hauteur à partir d'un âge donné).

Au stade actuel, l'utilisation du cumul sur un nombre maximum d'années (au-delà de la $5^{\circ}$ ) des pousses annuelles comme critère prédicteur du volume nous a conduit à étudier à une échelle plus fine la croissance en hauteur du Pin maritime. Ce caractère a ainsi été décomposé au niveau annuel, puis au niveau interannuel. Au niveau annuel, auquel, se limite cette étude, le Pin maritime peut réaliser plusicurs cycles successifs d'accroissement. Un rappel bibliographique précisera la notion de polycyclisme.

La présente contribution, début d'une étude plus générale, se propose d'analyser l'influence du rythme annuel sur la croissance en hauteur. Comment s'exprime le polycyclisme chez ie Pin maritime, quelle est l'importance des facteurs génétiques et environnementaux qui les conditionnent, quelle est son utilisation possible en sélection?

\section{2. - Rappel bibliographique}

La croissance annuelle de nombreux conifères est caractérisée par plusieurs cycles successifs d'accroissements. Certains pins font régulièrement plusieurs cycles (Pinus palustris) durant une année. D’autres réalisent occasionnellement un second ou un troisième cycle durant la saison de végétation. Tel est le cas du Pin maritime.

\section{Sur le plan morphologique}

Chaque pousse correspondant à un cycle est précédée par un verticille. Quand celui-ci n'est pas formé, le début d'un nouveau cycle est caractérisé sur la tige 
principale par une zone d'une dizaine de centimètres dépourvue d'aiguille. Ces caractéristiques morphologiques permettent d'individualiser aisément les différentes pousses.

\section{Sur le plan morphogénétique}

Dans une revue générale consacrée au développement morphogénétique du tronc chez les pins, R. LANNER (1976) effectuc une classification schématique des modalités de croissance.

- croissance fixe : élongation des articles préformés dans le bourgeon. Dans tout ce qui suit le terme article désigne toute ébauche d'éléments se trouvant sur la tige d'un pin (écaille «stérile», brachyblaste, auxiblaste, conelet). d'articles.

- croissance libre : élongation due à l'initiation et au développement simultané

En ce qui concerne le polycyclisme, cette vue schématique regroupe en fait des réalités différentes. En particulier la croissance fixe de la seconde pousse peut se caractériser par l'élongation d'articles préformés dans le bourgeon de l'année même ou de l'année précédente.

Par exemple, chez Pinus banksiana (Lanner, 1970) la réalisation d'un second cycle lors de l'année n peut être le résultat d'un développement précoce d'un bourgeon qui serait resté dormant jusqu'au printemps de lannée $n+1$, mais qui s'est allongé durant l'été de l'année n parce que les conditions climatiques étaient favorables. Par contre, chez Pinus contorta, le second cycle correspond au développement d'un bourgeon d'hiver, dans lequel les articles des deux cycles se sont formés à l'automne de l'année $\mathrm{n}-1$.

La croissance libre d"une seconde pousse se produit à partir de primordium sur le bourgeon de la saison de végétation et se réalise l'année même alors que la pousse primaire s'est déjà allongée (Pinus elliottii, Lanner, 1976). Elle est consécutive à une croissance fixe du premier cycle, entièrement prédéterminé dans le bourgcon formé laatomne précédent.

Enfin croissance libre et croissance fixe peuvent être incriminćes dans l'cxpression du polycyclisme notamment chez les espèces réalisant plusicurs secondes pousses. Chez Pinus elliottii ces dernières suivent toutes une croissance libre.

Cependant chez Pinus echinata ou Pinus rigida, la première d'entre elles est déjà préformée dans le bourgeon. En d'autres termes la croissance libre du troisième ou quatric̀me cycle suit la croissance fixe des deux premiers (LANNER, 1976).

En ce qui concerne le Pin maritime, les quelques observations qui ont été faites montrent que la croissance en hauteur de cette espèce ne saurait correspondre exclusivement à l'un ou à l'autre de ces mécanismes. Les observations faites sur la genèse et le développement de la seconde pousse sont contradictoires. Pour DAviD (1968), à la fin de l'hiver précédant la saison de végétation, les deux pousses se trouvent déjà différenciées dans le bourgeon. «La seule différence existant entre les deux types de bourgeons (bourgeon à une pousse et bourgeon à deux pousses) résulte du fait que l'anneau initial demeure actif pendant tout lhiver chez les sujets à deux pousses ». Cette observation va à l'encontre de celle de MAvge e' all. (1976) : dans 
les essais de fertilisation azotée les fréquences d'apparition de seconds cycles sont augmentées par une application d'azote au printemps. Enfin, Debazac (1963) note que les accroissements primaires et secondaires se développent presque simultanément avec un très faible décalage.

Que l'initiation et l'élongation des unités élémentaires constituées par l'espace séparant deux articles successifs («Stem unit») se fassent simultanément ou non, il apparaît clairement que pour les pins de la zone tempérée l'accroissement total est beaucoup plus lié au nombre d'unités initiées qu'à l'élongation de celles-ci (I_ANNER, 1976; CANNEL, 1976) $\left(^{*}\right)$. En clair, cela signifie que pour les espèces où l'initiation dans le bourgeon se fait à l'été $\mathrm{n}-1$ la pousse de l'année $\mathrm{n}$ dépend autant sinon plus des conditions climatiques de lannée $\mathrm{n}-1$ que de celles de l'année $n$.

\section{Sur le plan courbe de croissance}

L'accroissement en hauteur du Pin maritime est caractérisé par deux pics successifs (l'un en avril-mai, l'autre en août), le second étant nettement moins accentué que le premier (CASTAING \& Illy, 1966). Il faut noter cependant qu'un pic de croissance ne correspond pas à un cycle de croissance au sens morphogénétique du terme. En effet, les autcurs remarquent «qu'il n'apparaît pas de liaison entre la formation de verticilles et la croissance en hauteur». On peut faire remarquer à ce sujet l'ambiguïté du langage courant qui tend à désigner le second cycle de croissance par «pousse d'août».

Par contre chez d'autres espèces (Pinus rigida Mill., Pinus echinata Mill., TEPPER, 1963), il y a analogie entre le second pic de croissance et le développement d'une seconde pousse.

Chez ces mêmes espèces comme chez d'autres (Pinus banksiana Lamb.) les individus polycycliques ont une saison de végétation plus longue que les individus monocycliques (RudolpH, 1964).

\section{3. - Matériel et mesures}

Des mesures de hauteurs totales annuelles ont été faites sur plusieurs dispositifs expérimentaux : le niveau des verticilles correspondant aux différentes années a été noté. Dans le cas d'accroissements annuels à plusieurs pousses la hauteur correspondant à la base de chacune a été relevée. Toutes ces mesures ont été faites simultanément et rétrospectivement à l'aide d'un décamètre. Les erreurs de mesures dues à la répétition de mesures de hauteur totale à origine non fixée sont ainsi supprimées. On dispose ainsi facilement des accroissements du premier cycle (accroissement primaire), du second cycle (accroissement secondaire) et des accroissements totaux annuels.

(*) A une échelle plus macroscopique, chez des espèces qui font régulièrement plusieurs cycles de végétation (Pinus palustris) t'accroissement total est plus corrélé au nombre de cycles qu'à l'élongation de ceux-ci (Ali.en, Scarbrough, 1970). 


\subsubsection{1. (Verger à graines de clones de Saint-Pee-sur-Nivelle}

Ce verger à graines est composé de 5 tranches annuelles correspondant chacune à une année de greffage (de 1962 à 1966). Les copies d'un même clone sont réparties au hasard à l'intérieur d'une tranche ce qui confère à celle-ci la structure d'un dispositif mono-arbre. 155 clones font partie du dispositif; le nombre de copies par clone est variable (de 1 à 36 ).

Très peu de clones sont communs à deux tranches, l'interprétation peut donc être faite selon un schéma hiérarchique (premier niveau : tranche, second niveau : les clones). Les mesures ont été faites sur toutes les copies.

\subsubsection{0. (Test de descendances de pleins frères, Rousset)}

Ce test de descendances a été mis en place en 1970. Il comprend 169 familles appartenant à quatre plans de croisements dont un plan de croisement factoriel (HinkelmanN \& Stern, 1960) a été utilisé dans cette étude. Il est composé de familles issues de croisements entre 4 pères et 21 mères. Le dispositif est un lattice équilibré $13 \times 13$ à 7 répétitions. Chaque parcelle unitaire comprend neuf plants.

Les mesures ont été faites sur un échantillon systématique de 4 plants par parcelle unitaire. Une fertilisation azotée a été appliquée au printemps 1976.

\subsubsection{1. (Test de descendances de demi-frères, Saint-Alban)}

Installé en lande semi-humide en automne 1965, ce test comprend 100 familles de demi-frères issus de pollinisation "d'arbres plus» en forêt. Le dispositif est un lattice triple $10 \times 10$ répété 3 fois. Chaque parcelle unitaire contient 10 plants. Les mesures ont été faites sur un échantillon systématique dc 3 plants par parcelle unitaire.

\subsubsection{2. (Test de descendances de demi-frères, Soustons)}

Ce test est contemporain du 2.44.3.1. et a été installé en dune. Il comprend 100 familles de demi-frères communes au 2.44.3.1. Le dispositif est un lattice triple $10 \times 10$ répété deux fois. Chaque parcelle unitaire contient 10 plants. Les mesures ont été faites sur un échantillon systématique de 4 plants par parcelle unitairc.

Le tableau 1 représente les années de végétation pour lesquelles les mesures ont été faites.

Au plan écologique, Le Rousset et Saint-Alban se trouvent en lande semihumide qui correspond aux conditions optimales de développement du Pin maritime. Soustons se trouve en dune, à la limite avec la lande. Schématiquement ce dernier milieu se caractérise surtout par la sécheresse estivale : le sol est squelettique et la nappe descend à un niveau relativement profond en été. Enfin, le verger à graines de Saint-Pee-sur-Nivelle est situé en dehors du massif forestier et se trouve dans le Pays Basque. 


\section{4. - Résultats}

\section{1. - Constitution de la pousse annuelle}

Les résultats globaux issus de l'ensemble des dispositifs sont rassemblés dans le tableau 1. Outre les pousses primaires et secondaires, deux caractéristiques liées au polycyclisme y figurent également : le rapport de la pousse secondaire sur la pousse totale et la fréquence d'individus ayant une seconde pousse

\section{Importance du polycyclisme}

En moyenne la pousse secondaire représente 11 p. 100 de l'accroissement annuel, ce chiffre pouvant varier de 31 p. 100 à 3 p. 100 . Cette moyenne est calculée sur l'ensemble des individus y compris ceux n'ayant pas réalisé une seconde pousse : ceci explique la redondance des deux composantes du polycyclisme. La valeur de 11 p. 100 est importante quand on sait que le coefficient de variation phénotypique de l'accroissement total en hauteur est de cet ordre de grandeur $(10 \mathrm{p}$. 100) : ce chiffre pourrait justifier l'utilisation du polycyclisme comme critère de sélection pour la croissance.

\section{Influence de l'âge}

Les dispositifs 2.44.10. et 2.44.3.1. se trouvent dans les mêmes conditions stationnelles. Par contre les plants ont une différence d'âge de 4 ans. La comparaison des valeurs de II/ $(I+$ II) et de F montre que l'effet âge joue un rôle important dans l'expression du polycyclisme. En effet, ces valeurs varient du simple au double en passant du second au premier dispositif.

A l'intérieur de chaque test de descendances, on observe par ailleurs une certaine décroissance de II/(I + II) et de F avec l'âge.

En valeur absolue, cette évolution est quelque peu infirmée par les résultats du test clonal (5.44.01.). Sans doute, les conditions écologiques particulières de son implantation masquent-elles l'expression de l'âge. D'autre part il n'est pas exclu que le polycyclisme soit affecté par les effets de topophysis.

\section{Influence de la station, du milieu}

La comparaison de la croissance lors d'années homologues dans les deux tests 2.44.3.1. et 2.44.3.2. montre que le polycyclisme s'exprime d'autant plus que le milieu est moins sec. Ceci est vrai autant pour l'accroissement secondaire que pour l'aptitude au polycyclisme exprimée en pourcentage de pousses d'août. Dans un milieu souffrant de sécheresse estivale (2.44.3.2.) l'accroissement secondaire ne représente guère que 3 à 6 p. 100 de l'accroissement total alors que ces chiffres pour les mêmes populations et les mêmes années varient de 4 p. 100 à 16 p. 100 en lande humide. Par ailleurs l'application d'une fertilisation azotée au printemps augmente la fréquence des pousses d'août de la même année (2.44.10., année 1976). 


\section{TABleau 1}

Moyentes des différents accroissements et fréquences d'expression du polycyclisme Annual means of height increments and frequencies of trees with second shoot

\begin{tabular}{|c|c|c|c|c|c|c|c|c|}
\hline Test & $\begin{array}{l}\text { Année de } \\
\text { végétation }\end{array}$ & Caractère & 71 & 72 & 73 & 74 & 75 & 76 \\
\hline \multirow{4}{*}{$\begin{array}{l}\dot{\delta} \\
\dot{J} \\
\text { in }\end{array}$} & \multirow{4}{*}{$\begin{array}{c}7^{\circ} * \\
\grave{a} \\
12^{\circ}\end{array}$} & I & 70.6 & 67.4 & 61.9 & 74.9 & 60.2 & \\
\hline & & II & 9.7 & 13.1 & 8.7 & 4.0 & 2.9 & \\
\hline & & $11 /(I+I I)$ & 12.1 & 16.2 & 12.3 & 5.1 & 4.6 & \\
\hline & & $\mathrm{F}$ & 64.7 & 84.3 & 60.8 & 38.2 & 20.6 & \\
\hline \multirow{4}{*}{$\begin{array}{l}\dot{0} \\
\stackrel{+}{\dot{J}} \\
\stackrel{i}{i}\end{array}$} & \multirow{4}{*}{$\begin{array}{l}5^{\circ} \\
\mathrm{a} \\
8^{\circ}\end{array}$} & I & & & 37.9 & 66.2 & 74.4 & 92.7 \\
\hline & & II & & & 17.4 & 11.9 & 10.5 & 24.6 \\
\hline & & $I I /(I+I I)$ & & & 31.4 & 15.2 & 12.3 & 21 \\
\hline & & $\mathrm{F}$ & & & 69.7 & 42.5 & 33.8 & 79.3 \\
\hline \multirow{4}{*}{$\begin{array}{l}\dot{-} \\
\dot{m} \\
\dot{a} \\
\dot{i}\end{array}$} & \multirow{4}{*}{$\begin{array}{c}7^{\circ} \\
\grave{a} \\
12^{\circ}\end{array}$} & I & 90 & 89.5 & 75.2 & 94.9 & 76.7 & 83.9 \\
\hline & & II & 16.1 & 14.7 & 14.6 & 5.8 & 3.7 & 5.2 \\
\hline & & $\mathrm{II} /(\mathrm{I}+\mathrm{II})$ & 15.2 & 14.1 & 16.2 & 5.8 & 4.6 & 5.9 \\
\hline & & F & 53.2 & 54.6 & 45.8 & 22.1 & 11.7 & 35.8 \\
\hline \multirow{4}{*}{$\begin{array}{l}\stackrel{i}{~} \\
\stackrel{m}{j} \\
\stackrel{j}{i}\end{array}$} & \multirow{4}{*}{$\begin{array}{c}9^{n} \\
\grave{a} \\
12^{\circ}\end{array}$} & I & & & 61.0 & 80.9 & 82.9 & 80.1 \\
\hline & & II & & & 3.4 & 3.8 & 2.9 & 4.9 \\
\hline & & $\mathrm{II} /(\mathrm{I}+\mathrm{II})$ & & & 5.2 & 4.5 & 3.4 & 5.7 \\
\hline & & $\mathrm{F}$ & & & 23.6 & 21.3 & 15.5 & 33 \\
\hline
\end{tabular}

I : Moyenne des pousses primaires $(\mathrm{cm})$.

II : Moyenne des pousses secondaires.

II $/(\mathrm{I}+\mathrm{II})$ : Rapport des moyennes de pousses secondaires sur les moyennes de pousses totales (en pourcentage).

F : Fréquence des arbres ayant réalisé une pousse secondaire (en pourcentage).

$\because$ : après le greffage.

I : Annual mean of first shoot increment $(\mathrm{cm})$.

II : Annual mean of second shoot increment $(\mathrm{cm})$.

II / I + II $):$ Ratio of the mean of second shoot increment on the mean of total annual shoot increment.

F: Frequency 'of trees with second shoot.

$\because$ : After grafting.

En résumé, les deux facteurs externes qui déterminent principalement l'expression du polycyclisme sont les variations climatiques annuelles [dans chaque dispositif expérimental, les valeurs de $\mathrm{I} /(\mathrm{I}+\mathrm{II})$ peuvent varier du simple au double dannéc 
en année]. L'autre facteur est le milieu physique, les valeurs de $\mathrm{I} /(\mathrm{I}+\mathrm{II})$ du 2.44.3.2. sont nettement différentes de celles des autres dispositifs expérimentaux. Enfin, l'âge physiologique joue également un rôle important.

\section{2. - Expressions du polycyclisme}

Niveau annuel

Les histogrammes annuels des pousses secondaires (cf. figure 1) reflètent le caractère «tout ou rien» de l'expression du polycyclisme. Une interprétation n'est possible qu'en le décomposant en deux sous caractères :

- la fréquence des pousses secondaires (F) exprimant l'aptitude au polycyclisme (caractère discret) ;

- l'élongation des pousses secondaires mesurée uniquement sur les individus layant manifestée (E). La distribution des élongations secondaires dans cette souspopulation est celle d'une loi normale et permet de ce fait de comparer les différentes pousses, primaires, secondaires et totales entre elles (voir figure 1).

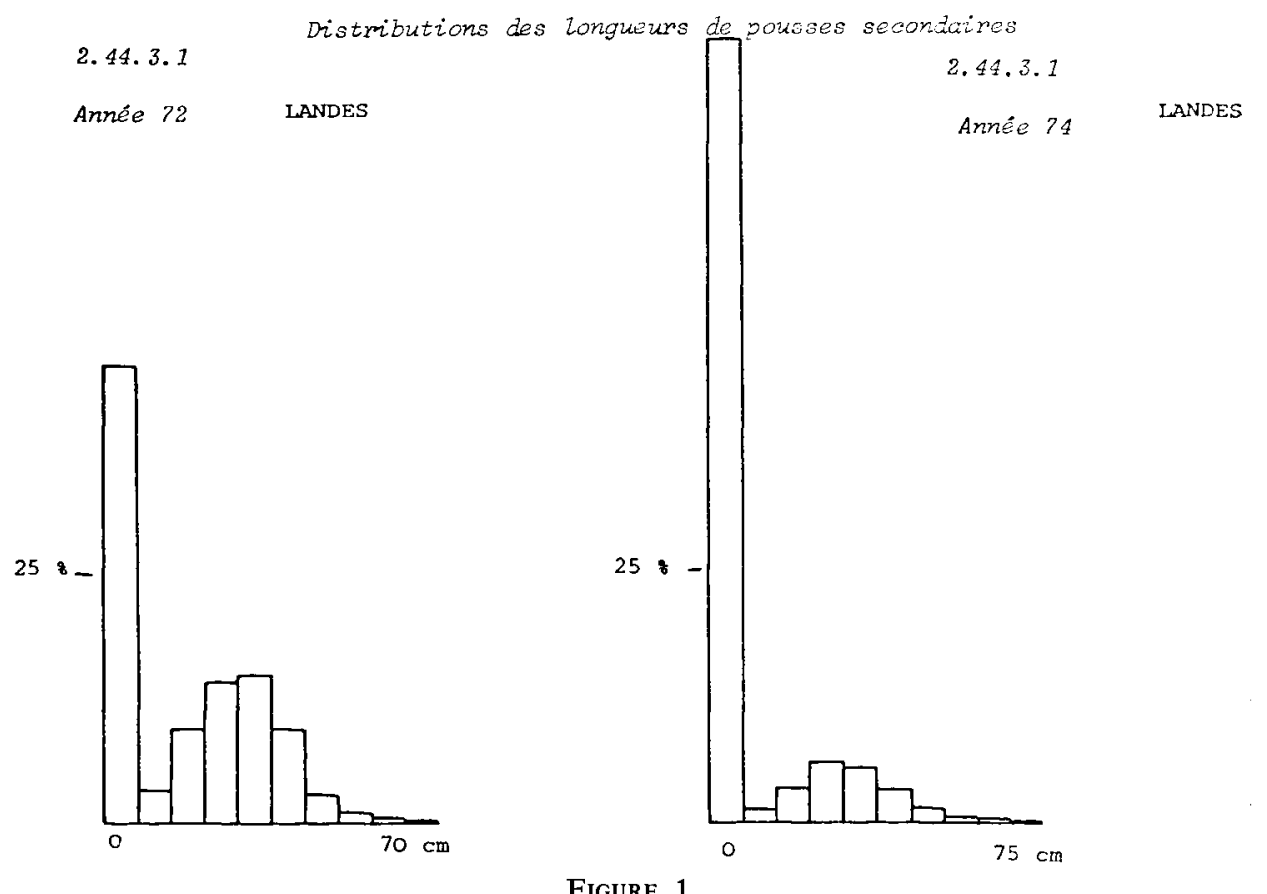

Distributions des longueurs de pousses secondaires

Distributions of second shoot increments 
$\mathrm{E}$ et $\mathrm{F}$ sont des composantes du polycyclisme tout à fait indépendantes. En effet, les coefficients de corrélation au niveau familial entre $E$ et $F$ ne sont que très rarement significatifs. Par ailleurs, bien que le nombre d'années ne soit pas suffisant pour estimer avec précision les coefficients de corrélation environnementaux, les moyennes annuelles de $\mathrm{E}$ et $\mathrm{F}$ (cf. tableau 2) montrent que les types d'expression du polycyclisme sont divers (année à fréquence élevée et à élongation élevée, année à fréquence élevée et à élongation faible, etc.). Apparemment que ce soit au niveau génétique ou environnemental de l'expression du polycyclisme, il n'existe pas de compensation ou d'équilibre entre la fréquence de sa réalisation et l'élongation de la seconde pousse.

La variabilité interannuelle élevée de II/(I + II) dans le tableau 1 est essentiellement liée à celle de F (cf. tableau 2). En effet, l'élongation secondaire quand elle s'exprime représente une proportion relativement stable d'année en année de l'élongation totale $(25 \%$ de l'élongation totale dans les landes et $19 \%$ dans les dunes).

\section{Tableau 2}

Moyennes de $E, F$ et corrélation entre $E$ et $F$ Annual mean of $E, F$ and correlation coefficient between $E$ and $F$

2.44.3.1. - Landes

\begin{tabular}{l|c|c|c|c|c|c}
\hline Année . . & 71 & 72 & 73 & 74 & 75 & 76 \\
\hline $\mathrm{E} \ldots \ldots \ldots$ & 25.8 & 23.9 & 30.5 & 24.0 & 30.4 & 15.5 \\
\hline $\mathrm{F} \ldots \ldots \ldots$ & 53.2 & 54.6 & 45.8 & 22.1 & 11.7 & 35.8 \\
\hline $\mathrm{r}(\mathrm{E}, \mathrm{F}) \ldots \ldots$ & .15 & .05 & .16 & .19 & .08 & .10 \\
\hline $\mathrm{D} \ldots \ldots \ldots$ & 21.8 & 17.9 & 28.7 & 9.7 & 30.5 & 8.7 \\
\hline
\end{tabular}

2.44.3.2. - Dunes

\begin{tabular}{|c|c|c|c|c|}
\hline Année .... & 73 & 74 & 75 & 76 \\
\hline$\ldots \ldots \ldots$ & 19.2 & 20.3 & 20.0 & 16.4 \\
\hline$F \ldots \ldots \ldots$ & 23.6 & 21.3 & 15.5 & 33.0 \\
\hline$r(E, F) \quad \ldots$ & $.30 \%$ & .05 & .15 & -.13 \\
\hline D & 15.8 & 4.9 & 7.3 & 8.7 \\
\hline
\end{tabular}

E : Elongation secondaire exprimée en pourcentage de l'élongation totale.

$\mathrm{F}$ : Fréquence des pousses secondaires exprimée en pourcentage.

$r(E, F)$ : Coefficient de corrélation entre moyennes familiales de $E$ et de $F$.

D : Différence entre élongations totales des polycycliques et des monocycliques exprimée en pourcentage de l'élongation totale moyenne de l'année.

E : Second shoot increment expressed in percentage of the total shoot increment.

$\mathrm{F}:$ Frequency of trees with second flush.

r $(E, F)$ : Correlation coefficient between family mean values of $E$, and $F$.

D : Difference between total annual shoot increment of polycyclic and monocyclic trees expressed in percentage of the mean total annual shoot increment. 
Niveau interannuel

Au niveau interannuel, l'étude des fréquences permet de réaliser une partition de l'ensemble des individus en trois sous-ensembles :

(A) celui des polycycliques «stricts», manifestant chaque année une seconde pousse ;

(B) celui des monocycliques «stricts» ne manifestant aucune pousse secondaire au cours des 4 ou 6 années;

(C) celui des polycycliques ou monocycliques occasionnels ne réalisant qu'exceptionnellement, lors d'une ou de plusieurs années, une seconde pousse.

Les tableaux de fréquence du nombre de seconds cycles témoignent de la différence de l'expression du polycyclisme d'un milieu à l'autre (cf. figure 2). En landes, la distribution du nombre de pousses secondaires n'est pas comparable à celle des dunes. Dans le premier cas, la majorité de l'effectif est constituée de polycycliques occasionnels, dans le second de monocycliques stricts : toutefois, la fréquence de polycycliques stricts reste constante d'un milieu à l'autre (fig. 2).

\subsubsection{Landes}

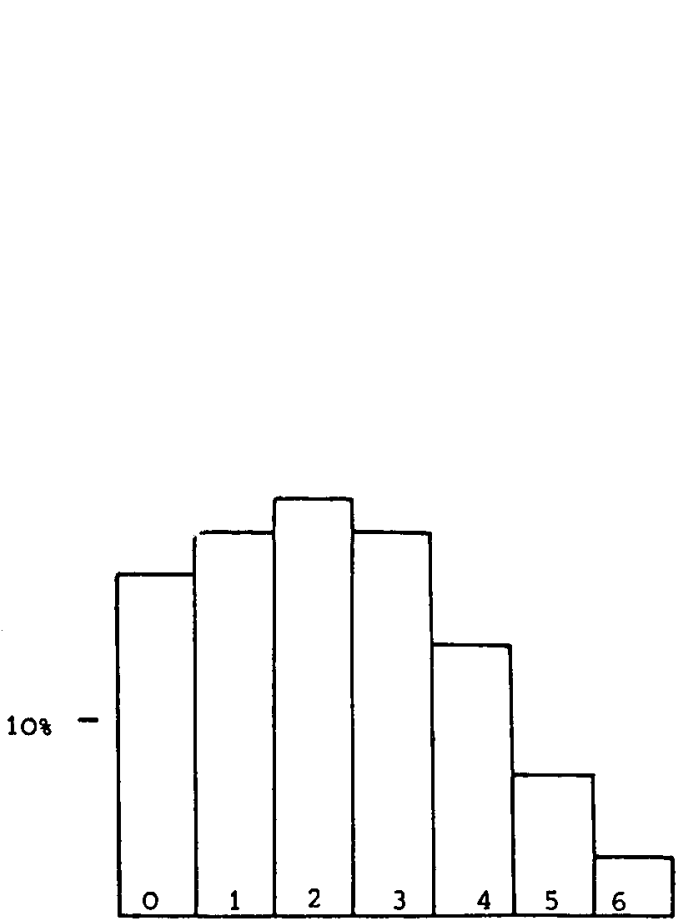

2.44.3.2. Dunes

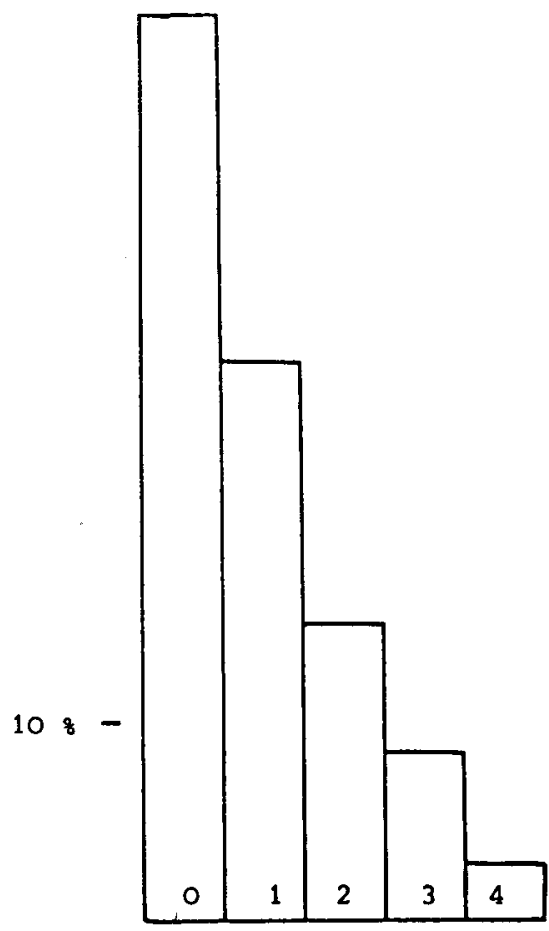

Figure 2

Distributions du nombre de pousses secondaires réalisées durant les $n$ années d'observation Distributions of the number of second shoots expressed during $n$ years 
Enfin, raccroissement total des polycycliques d'une année donnée est toujours supérieur à celui des monocycliques. La différence, toujours significative, peut représenter de 9 à 30 p. 100 de la moyenne générale de l'élongation totale (cf. tableau 2), dans les landes et de 5 à 16 p. 100 dans les dunes.

\section{3. - Modalités de croissance liées au polycyclisme}

Les différents accroissements (primaires, secondaires et totaux) des individus appartenant aux sous-ensembles (A) (B) et (C) sont représentés sur les figures 3 (a ct b). Le relatif parallélisme des courbes $1-4,6-3,2$ et 5 et la stabilité du classement des moyennes des différents sous-ensembles conduisent à penser qu'il existe un type de croissance lié au polycyclisme et un type de croissance lié au monocyclisme. Pour la clarté de l'exposé, le sous-ensemble B a été partagé chaque année en sous-population polycyclique (polycycliques des polycycliques occasionnels) et monocycliques (monocycliques des polycycliques occasionnels).

En d'autres termes, la courbe 2 joint les moyennes des accroissements primaires des individus ayant réalisé un second cycle en 1971, à ceux (différents des précédents) ayant réalisé un second cycle en 1972, etc. Par contre, les courbes 4, 5, 6 correspondent à des populations identiques d'année en année.

- La réalisation d'une seconde pousse confère toujours un avantage très net aux polycycliques. Cet avantage demeure constant lors de toutes les années chez les polycycliques stricts. Par contre, l'avantage acquis chez les polycycliques occasionnels lors d'une année $n$, supérieur à celui des précédents pour cette année, est en partie annulé l'année suivante par la fréquence non négligeable d'individus ne réalisant pas de seconde pousse l'année $(\mathrm{n}+1)$ ou les années suivantes. En effet, l'accroissement primaire (total) des monocycliques des polycycliques occasionnels (C) est toujours inférieur à celui des monocycliques stricts (B) (cf. fig. 4).

- D'une manière plus générale, sur la figure 3 (a) les courbes $6,3,2$ et 5 sont pratiquement parallèles. La croissance primaire chez les monocycliques ou polycycliques (stricts et occasionnels) obéit donc à des modalités comparables.

Les observations faites sur le dispositif 2.44.3.1. (figure 3 a) demeurent valables en grande partie pour le dispositif 2.44.3.2. (3 b). Cependant, dans ce dernier cas les différences entre élongation totale des monocycliques et des polycycliques stricts sont relativement minimes. Par analogie, ce résultat permet d'interpréter ces mêmes différences en 1974 et en 1976 au dispositif 2.44.3.1. Il est probable que lors de ces années, les arbres ont souffert de sécheresse estivale en landes.

\section{4. - Corrélations entre accroissements}

Dans le dispositif 2.44.3.1., les coefficients de corrélations génétiques environnementaux et phénotypiques entre accroissements ont été estimés pour les années où la fréquence d'expression des pousses secondaires par famille était suffisante. 
2.44.3.1. - Landes

(a)

\section{1}

72

73

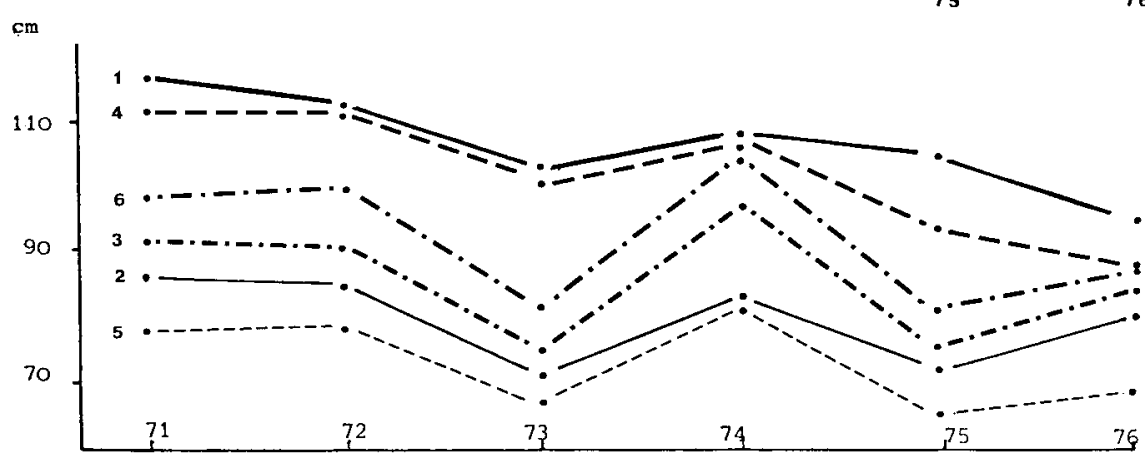

2.44.3.2. Dunes

(b)

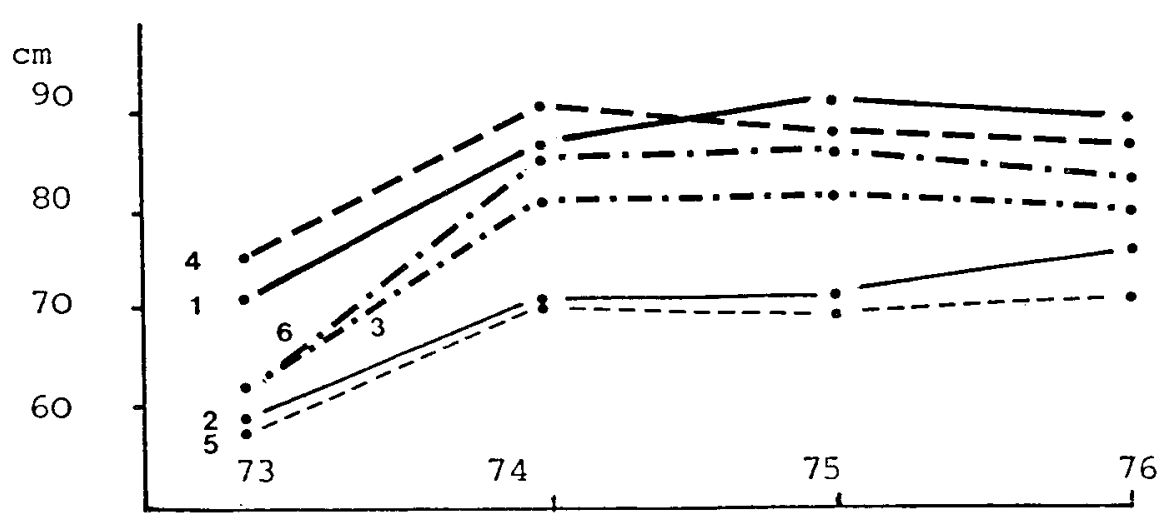

Figure 3

Moyennes des différents accroissements des individus polycycliques stricts, monocycliques stricts et polycycliques occasionnels

Means of different shoot increments of only polycyclic, only monocyclic and occasional polycyclic trees

1 : Elongation totale des polycycliques des polycycliques occasionnels (C).

2 : Elongation primaire des polycycliques des polycycliques occasionnels (C).

3 : Elongation totale (primaire) des monocycliques des polycycliques occasionnels (C).

4 : Elongation totale des polycycliques stricts (A).

5 : Elongation primaire des polycycliques stricts $(\mathrm{A})$.

6 : Elongation totale (primaire) des monocycliques stricts (B).

1 : Total shoot increment of the polycyclic trees from the occasional polycyclic population (C).

2 : First shoot increment of the polycyclic trees from the occasional polycyclic population (C).

3 : First (or total) shoot increment of the monocyclic trees from the occasional polycyclic population (C).

4 : Total shoot increment of the strictly polycyclic trees (A).

5 : First shoot increment of the strictly polycyclic trees (A).

6 : First (or total) shoot increment of the strictly monocyclic trees (B). 
Ces estimations portent sur la population d'individus ayant exprimé une seconde pousse en 1971, 1972, 1973. Elles se trouvent dans le tableau 3. Les erreurs standard ont été estimées par la méthode de Tallis (1959).

- Accroissements primaires et secondaires sont liés très étroitement à l'accroissement total et aux trois niveaux; ceci est dû pour une bonne part à une certaine autocorrélation entre les caractères (les pousses primaires et secondaires étant comprises dans les pousses totales).

- A l'opposé, les déterminismes internes et externes des élongations primaires et secondaires ne suivent pas un schéma bien défini. Si aux plans environnemental et phénotypique, la liaison est faiblement négative voir nulle, au plan génétique, elle est successivement fortement positive, négative puis nulle. Tout se passe comme si les facteurs endogènes liés à l'élongation de la seconde pousse changcaient d'année en année.

Tableau 3

Coefficients de corrélation entre accroissements Correlation coefficients between different shoot increments

\begin{tabular}{|c|c|c|c|c|}
\hline & & 1971 & 1972 & 1973 \\
\hline \multirow{3}{*}{ I, II } & $r_{A}$ & $.86 \pm .41$ & $-.55 \pm .40$ & $.08 \pm .33$ \\
\hline & $\mathrm{r}_{\mathrm{n}}$ & $-.07 \pm .03$ & $-.17 \pm .11$ & $-.17 \pm .42$ \\
\hline & $r_{p}$ & $-.04 \pm 02$ & $-.18 \pm .13$ & $-.15 \pm .34$ \\
\hline \multirow{3}{*}{ II, $(\mathrm{I}+\mathrm{II})$} & $r_{A}$ & $.97 \pm .10$ & $.81 \pm .46$ & $.83 \pm .11$ \\
\hline & $r_{k}$ & $.51 \pm .05$ & $.43 \pm .23$ & $.56 \pm .05$ \\
\hline & $r_{P}$ & $.53 \pm .05$ & $.43 \pm .20$ & $.57 \pm .05$ \\
\hline \multirow{3}{*}{$\mathrm{I},(\mathrm{I}+\mathrm{II})$} & $r_{A}$ & $.95 \pm .11$ & $.65 \pm .10$ & $.62 \pm .19$ \\
\hline & $\mathrm{r}_{\mathrm{I}}$ & $.82 \pm .09$ & $.81 \pm .30$ & $.72 \pm .14$ \\
\hline & $\mathrm{r}_{\mathbf{r}}$ & $.82 \pm .08$ & $.81 \pm .36$ & $.72 \pm .12$ \\
\hline
\end{tabular}

$\mathrm{r}_{\mathrm{A}}=$ Coefficient de corrélation génétique.

$\mathrm{r}_{\mathrm{F}}=$ Coefficient de corrélation due au milieu.

$r_{P}=$ Coefficient de corrélation phénotypique.

I, II, I + II sont définis dans le tableau 1.

$r_{A}$ : Genetic correlation coefficient.

$r_{\mathrm{E}}$ : Environmental correlation coefficient.

$r_{1}$ : Phenotypic correlation coefficient.

I, II, I + II are defined in table 1.

Ces résultats sont complétés par les corrélations phénotypiques estimées sur les seuls individus ayant réalisé une seconde pousse chaque année (polycycliques stricts) : au plan phénotypique la liaison entre les deux pousses reste souvent négative 
(tableau 4). Une autre illustration de ces résultats est donnéc dans la figure 4. Elle représente l'évolution de la croissance primaire des polycycliques occasionnels ayant réalisé un second cycle une année donnée, comparée à l'évolution de la croissance primaire des monocycliques stricts.

La réalisation d'un second cycle lors d'une année $\mathrm{n}$ peut affecter la croissance primairc de cette même année (polycycliques occasionnels en 1972, 1974, 1976), ou celle de l'année suivante, $\mathrm{n}+1$ (polycycliques occasionnels en 1971, 1973, 1975).

2.44.3.1. Landes

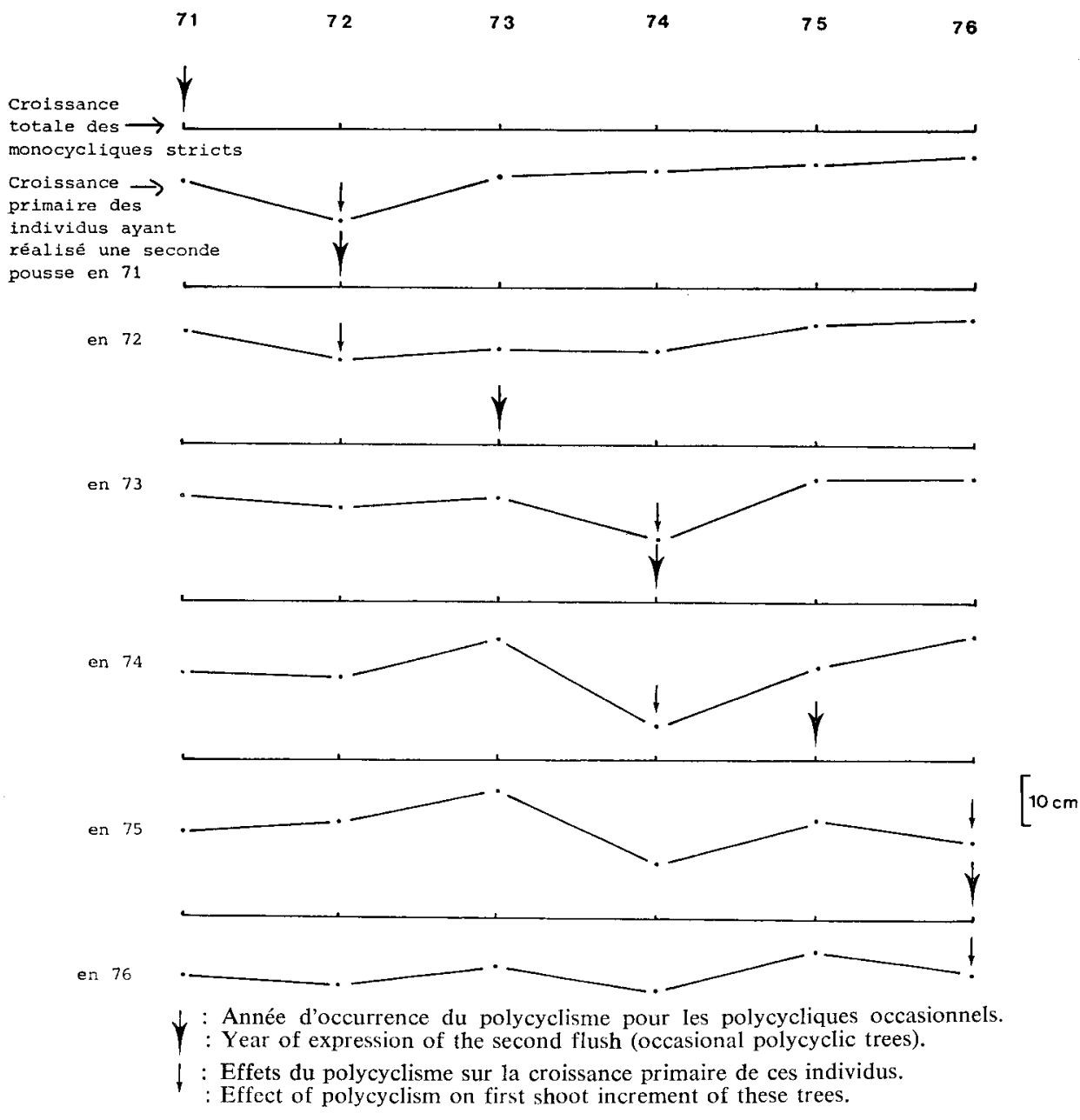

FIgure 4

Croissance primaire comparée des polycycliques occasionnels et des monocycliques stricts

First shoot increment of the occasionnal polycyclic trees compared with first shoot increment of the strictly monocyclic trees 
Si ce résultat apparaît clairement sur les quatre premières années, il est vérifié avec plus de nuance sur les deux dernières.

\section{5. - Paramètres génétiques}

Héritabilité et variance de dominance

Ces deux paramètres :

$$
h^{2}=\frac{\sigma^{2}{ }_{A}}{\sigma^{2}{ }^{\prime}} \text { et } \quad h_{11}=\frac{\sigma^{2}{ }^{2}}{\sigma^{2}{ }_{G}}
$$

ont été estimés à partir du plan de croisement $n^{n} 1$ du 2.44.10. (4 pères et 21 mères).

$\sigma^{2}{ }_{A}$ : variance additive.

$\sigma^{2}$ : : variance génotypique.

$\sigma_{\Gamma}^{2}$ : variance phénotypique.

Le plan de croisement du dispositif 2.44.10. a été interprété en utilisant le modèle d'HENDERSON III (SEARLE, 1971), analyse de variance non orthogonale à deux facteurs (aléatoires ou fixes) :

$$
Y_{i j k}=\mu+M_{i}+P_{j}+(M P)_{i j}+\varepsilon_{i j k}
$$

$P_{j} \quad$ : effet père $N\left(O, \sigma^{2}{ }_{p}\right)$.

$\mathrm{M}_{\mathrm{i}} \quad$ : effet mère $\mathrm{N}\left(\mathrm{O}, \sigma^{2}{ }_{\mathrm{m}}\right)$.

$(\mathrm{MP})_{\mathrm{ij}}$ : effet interaction mère $\times$ père $\mathrm{N}\left(\mathrm{O}, \sigma^{2}{ }^{2}{ }_{\mathrm{m}}\right)$.

$\varepsilon_{\mathrm{ijk}} \quad$ : effet individu $\mathrm{N}\left(\mathrm{O}, \sigma^{2}{ }^{2}\right)$.

Les variances correspondant aux différents effets sont estimécs en identifiant les sommes de carrés à leurs espérances et en résolvant le système d'équation ainsi obtenu :

$$
\begin{aligned}
& \mathrm{S}_{1}=(\mathrm{C}-\mathrm{M}) \sigma^{\ddot{2}}+\mathrm{K}_{1} \sigma^{2}{ }_{\mathrm{ml}}+\mathrm{K}_{1} \sigma^{2}{ }_{\mathrm{p}} \\
& \mathrm{S}_{2}=(\mathrm{C}-\mathrm{P}) \sigma^{2}{ }_{\mathrm{s}}+\mathrm{K}_{2} \sigma^{2{ }^{2}{ }_{m p}}+\mathrm{K}_{2} \sigma^{2}{ }_{\mathrm{m}} \\
& \left.S_{3}=(C-M-P+1) \sigma^{2}{ }_{8}+K_{3} \sigma^{2}{ }_{m p}\right) \\
& \mathrm{S}_{4}=(\mathrm{N}-\mathrm{C}) \sigma^{2}{ }^{2}
\end{aligned}
$$

$M$ et $P$ sont le nombre total de mères et de pères; $N$, nombre total dindividus; $\mathrm{C}$, nombre total de combinaisons mère $\times$ père et $\mathrm{K}_{1}, \mathrm{~K}_{2,}, \mathrm{~K}_{3}$ constantes liées au dispositif.

- En ce qui concerne $E$ (caractère continu), l'estimation des variances parentales et d'interaction mère $\times$ père étant faite, on peut appliquer le modèle génétique.

$\sigma^{2}{ }_{A}$ est estimée en pondérant les variances parentales par le nombre de degrés de liberté du parent correspondant :

$$
\begin{aligned}
& \hat{\sigma}^{2}{ }_{A}=4 \frac{(M-1){\widehat{\sigma^{2}}}_{\mathrm{m}}+(\mathrm{P}-1) \widehat{\sigma_{\mathrm{p}}^{2}}}{(\mathrm{M}-1)+(\mathrm{P}-1)} \\
& \hat{\sigma}^{2}{ }_{\mathrm{D}}=4{\widehat{\sigma^{2}}}_{\mathrm{mp}}
\end{aligned}
$$


Tableau 4

Coefficients de corrélation phénotypique entre accroissements primaires et secondaires polycycliques stricts

2.44.3.1. - Landes

Phenotypic correlation coefficients between first and second shoot increments (polycyclic trees only). 2.44.3.1.

\begin{tabular}{|c|c|c|c|c|c|c|}
\hline II & 1971 & 1972 & 1973 & 1974 & 1975 & 1976 \\
\hline 1971 & .04 & -.08 & -.21 & $.24^{*}$ & -.22 & -.11 \\
\hline 1972 & .05 & $-.31 * *$ & -.06 & -.08 & -.01 & .14 \\
\hline 1973 & -.10 & -.04 & -.03 & .03 & -.16 & $-.29 *$ \\
\hline $1974 \ldots \ldots$ & .04 & $-.26 *$ & $-.22-$ & .07 & $-.26 *$ & $-.29 *$ \\
\hline $1975 \ldots \ldots$ & -.08 & -.12 & $.30 * *$ & $-.24^{*}$ & .21 & -.06 \\
\hline 1976. & .20 & $-.50 * *$ & $-.40^{* *}$ & -.10 & .06 & $-.36^{* *}$ \\
\hline
\end{tabular}

- En ce qui concerne F (caractère discret), le modèle d'Henderson III est également utilisé pour l'interprétation du plan de croisement factoriel à combinaisons manquantes. Cependant, la variance d'un caractère «tout ou rien» regroupe d'une part une partie binomiale liée à l'échantillonnage et d'autre part une partie non binomiale ne dépendant pas de l'effectif. Au niveau d'une famille, la variance intra, $\sigma^{2}$; se décompose ainsi en variance binomiale $\left(V_{1}\right)$ et en variance liée aux différences entre individus $\left(\sigma^{* 2}{ }_{\mathrm{i}^{\prime}}\right)$ :

$$
\sigma^{2}{ }_{\varepsilon}=\mathrm{V}_{1,}+\sigma^{2}{ }^{\prime}
$$

Les trois premières sommes de carrés du systèmes sont calculées après transformation en Arc sin $V$.

Si $\mathbf{P}_{\mathrm{i} j}$ représente la fréquence d'expression du polycyclisme dans cette famille la variance binomiale a pour expression $P_{i j}\left(1-P_{i j}\right)$. En d'autres termes cette composante environnementale $\left(\mathrm{V}_{1}\right)$ est liée à l'expression génétique du polycyclisme de la famille ij. La transformation Arc sin $\vee$ permet de s'affranchir de cette dépen-

dance et $V_{1}$, a pour estimation $\frac{\mathrm{N}}{4(\mathrm{~N}-\mathrm{C})}$ (ELSTON R.C., 1977). $\sigma^{2}{ }_{\varepsilon}$ est estimée par $\mathrm{V}_{1,}$.

De la sorte $\sigma^{2}{ }_{F}$ est sous-estimée et les autres variances des composantes de la variance phénotypique estimées de la même manière que précédemment, sont surestimées. On peut évaluer les biais affectant les variances intervenant dans $h^{2}$ et $\sigma^{2}{ }_{1} / \sigma^{2}{ }_{\mathrm{G}}$ compte tenu du système d'équation précédent :

$$
\begin{aligned}
& \text { biais }\left(\sigma^{2}{ }_{A}\right)=.017 \sigma^{2}{ }^{\prime}{ }^{\prime} \\
& \text { biais }\left(\sigma^{2}{ }_{0}\right)=.173 \sigma^{2}{ }^{\prime}{ }^{\prime} \\
& \text { biais }\left(\sigma^{2}{ }_{G}\right)=.190 \sigma^{\sigma^{2}}{ }^{\prime}{ }^{\prime}
\end{aligned}
$$


Par contre, la variance phénotypique est sous-estimée :

biais $\left(\sigma^{2}{ }^{\prime}\right):-.948 \sigma^{2}{ }^{\prime}$,

Finalement, l'héritabilité est surestimée et le rapport $\sigma^{2}{ }_{1)} / \sigma^{2}{ }_{(x}$ est peu affecté par le biais.

\section{Elongation}

Bien que ces paramètres naient de signification que pour les populations auxquelles ils s'adressent, les différences de leurs estimations sur les différentes souspopulations (monocycliques, polycycliques et cumul) sont telles que l'on peut leur accorder une certaine crédibilité (cf. tableau 5).

\section{TABleau 5}

Paramètres génétiques

1. - Polycycliques stricts

Genetic parameters

\begin{tabular}{|c|c|c|c|c|c|c|c|c|}
\hline & & & & II (E) & & $I+I I$ & $\begin{array}{l}\text { Fré } \\
\text { pouss }\end{array}$ & $\begin{array}{l}\text { des } \\
\text { ût }(F)\end{array}$ \\
\hline & $\mathrm{h}^{2}$ & $h_{d l}$ & $\mathrm{~h}^{2}$ & $h_{d}$ & $h^{2}$ & $h_{d}$ & $\mathrm{~h}^{2}$ & $h_{d}$ \\
\hline $1973 \ldots \ldots \ldots$ & .22 & 0 & .14 & 0 & .21 & 0 & .07 & .69 \\
\hline$\ldots \ldots \ldots$ & .24 & 0 & .21 & 0 & .24 & 0 & .12 & .16 \\
\hline $1975 \ldots \ldots \ldots$ & .21 & 0 & .14 & .02 & .40 & 0 & .20 & .30 \\
\hline $1976 \ldots \ldots \ldots$ & .23 & 0 & .19 & 0 & .22 & 0 & .08 & .26 \\
\hline
\end{tabular}

$$
\begin{aligned}
& \mathrm{h}_{\mathrm{d}}=\frac{\sigma^{2}{ }_{\mathrm{D}}}{\sigma_{{ }^{2}{ }_{\mathrm{i}}}} \\
& \mathrm{h}^{2}=\frac{\sigma_{\mathrm{A}}^{2}}{\sigma_{\mathrm{P}}^{2}}
\end{aligned}
$$

\section{2. - Monocycliques stricts}

\begin{tabular}{c|c|c}
\hline & & \multicolumn{2}{|c}{ Elongations } \\
\cline { 2 - 3 } & $\mathrm{I}=\mathrm{I}+\mathrm{II}$ \\
\hline $\mathrm{h}$ & $\mathrm{h}$ \\
\hline $1973 \ldots \ldots$ & .16 & $\frac{\mathrm{h}_{\mathrm{d}}}{.01}$ \\
\hline $1974 \ldots \ldots$ & .24 & $\frac{0}{0}$ \\
\hline $1975 \ldots \ldots$ & .15 & $\frac{0}{0}$ \\
\hline $1976 \ldots \ldots$ & .12 & 0 \\
\hline
\end{tabular}

\begin{tabular}{|c|c|c|c|c|}
\hline & \multicolumn{4}{|c|}{ Elongations } \\
\hline & \multicolumn{2}{|c|}{$\mathbf{I}$} & \multicolumn{2}{|c|}{$\mathrm{I}+\mathrm{II}$} \\
\hline & $h^{2}$ & $\mathbf{h}_{\mathrm{d}}$ & $h^{2}$ & $\mathrm{~h}_{\mathrm{d}}$ \\
\hline $1973 \ldots \ldots$ & .16 & .19 & .09 & .43 \\
\hline $1974 \ldots \ldots$ & .14 & .35 & .12 & .14 \\
\hline $1975 \ldots \ldots$ & .16 & .19 & .21 & 0 \\
\hline $1976 \ldots$ & .16 & .31 & .19 & .04 \\
\hline
\end{tabular}

3. - Cumul : polycycliques + monocycliques

Les valeurs d'héritabilité estimées isolément sur les polycycliques et les monocycliques stricts sont supéricures à celles estimées sur le cumul des deux, à l'exception des années 1975 et 1976 pour la sous-population des monocycliques stricts. 
Par ailleurs, en ne considérant que les polycycliques, les héritabilités des pousses totales sont aussi fortes que celles des pousses primaires, ce qui est plus rarement le cas dans le cumul.

L’augmentation des héritabilités va de pair avec une diminution des variances de dominance qui deviennent nulles quand elles sont estimées séparément sur les deux sous-populations polycycliques et monocycliques.

\section{Au niveau annuel}

\section{Fréquence d'expression}

Au niveau d'une année donnéc, la réalisation dune seconde pousse est surtout contrôlée par les effets additifs des gènes (à part l'année 1973). Néanmoins les effets environnementaux sont tels que les valeurs d'héritabilités ne sont jamais élevées.

\section{All nivean interannuel}

L'héritabilité du nombre de pousses secondaires réalisćes durant $n$ années d'observations a pu être estimée à partir du dispositif 2.44.3.1. (cf. distribution de ce caractère fig. 2). Ce dernier a été choisi parce que n est plus élcvé que dans les autres dispositifs et de ce fait il est plus facile de rendre normale la distribution du caractère par une transformation logarithme. L'héritabilité est estimée par $h^{2}=4 t$ où $t$ est le coefficient de corrélation intra classe. Jugé de cette manière, le polycyclisme est fortement héritable $\left(h^{2} \simeq .44\right)$.

\section{Stabilité interannulelle}

Le maintien du classement des familles pour les deux caractères liés au polycyclisme est estimé par les coefficients de corrélation entre valeurs familiales :

-.- des fréquences d'expression de secondes pousses pour F;

- des moyennes d’élongations secondaires pour $\mathrm{E}$.

Pour ce qui concerne le dernier cas, les estimations des coefficients de corrélations sont biaisées par le fait que les effectifs familiaux (d`individus exprimant une seconde pousse) ne sont constants ni de famille à famille, ni d'année en année. La moyenne familiale de l'élongation secondaire est ainsi biaisée, et les corrélations sont sous-estimées. Quoiqu'il en soit, les résultats du tableau 6 montrent que la fréquence d'expression est bien plus stable que l'élongation sans que cette stabilité soit vraiment importante.

\section{Stabilité inter-station}

Elle est estimée de la même manière par les coefficients de corrélation entre valeurs familiales de $E$ et de $F$ entre les deux stations de landes (2.44.3.1.) et de dunes (2.44.3.2.). Les remarques précédentes s'appliquent également ici. Les résultats (tableau 7) sont eux aussi analogues : la stabiiité de l'aptitude au polycyclisme est nettement plus importante que celle de l'élongation. Cette dernière est pratiquement nulle : les mécanismes intervenant dans l'élongation secondaire en landes sont différents de ccux incriminés en dunes, et ne sont ni positivement ni négativement liés entre eux. 


\section{TABLIEAU 6}

Coefficients de corrélation entre valeurs annuelles familiales de l'élongation secondaire $(E)$ ot de l'expression du polycyclisme $(F)$

Correlation coefficients betwe'n family values of $E$ and $F$

\subsubsection{1. - Landes}

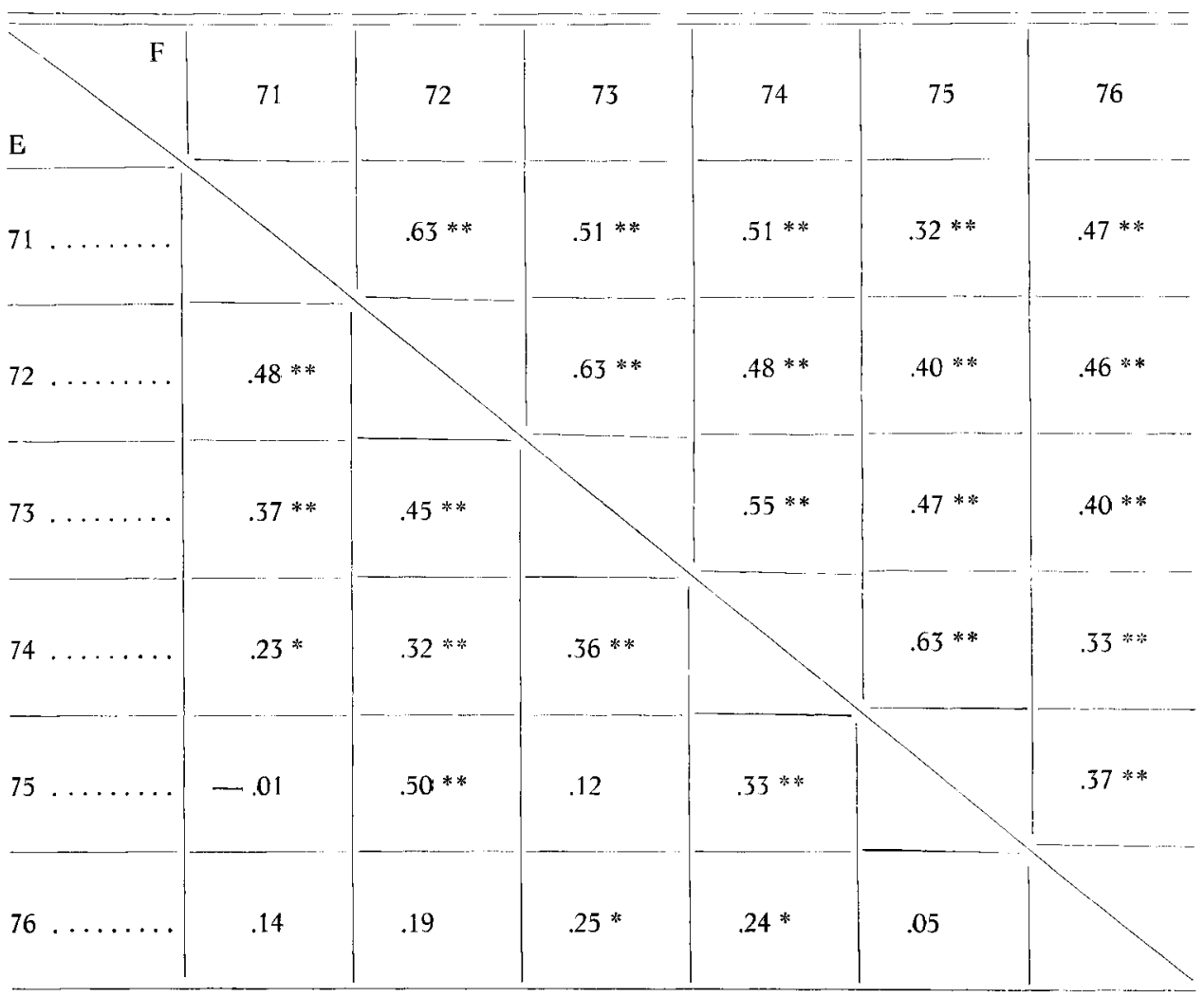

$*$ Test significatif au seuil de $5 \%$.

$*$ Test significatif au seuil de $1 \%$.

Un résultat intéressant et qui conforte l'hypothèse de la sécheresse en 1974 et 1976 au 2.44.3.1. est exprimé par les coefficients de corrélation élevés entre les valeurs familiales de $F$ pour les deux années 1974 et 1976 . En dautres termes, tout se passe comme si les mécanismes contrôlant l'expression du polycyclisme en landes lors des années 1974 et 1976 correspondaient à ceux qui agissent en dunes. 


\section{Tableau 7}

Coefficients de corrélation entre valeurs annuelles familiales au 2.44.3.I. et au 2.44.3.2. pour $E$ et $F$

Corrclations coefficients between annual family means in both designs (2.44.3.1 and 2.44.3.2.) for $E$ and $F$

Elongation secondaire (E)

\begin{tabular}{|c|c|c|c|c|}
\hline 2.44 .3 .1 .2 .44 .3 .2 & 1973 & 1974 & 1975 & 1976 \\
\hline 1973 & .15 & .12 & .03 & .15 \\
\hline$\ldots \ldots \ldots \ldots$ & .03 & .08 & -.01 & .09 \\
\hline$\ldots \ldots \ldots \ldots$ & -.06 & -.03 & -.02 & $一 .03$ \\
\hline 1976 & .14 & 一.01 & .03 & 一.08 \\
\hline
\end{tabular}

Fréquences des pousses secondaires $(F)$

\begin{tabular}{|c|c|c|c|c|}
\hline 2.44 .3 .1 .2 .44 .3 .2 & 1973 & 1974 & 1975 & 1976 \\
\hline$\ldots \ldots \ldots \ldots$ & $.35 * *$ & $.38^{* *}$ & $.20^{*}$ & $.46 * *$ \\
\hline 1974 & .18 & $.54 * *$ & .07 & $.39 * *$ \\
\hline$\ldots \ldots \ldots \ldots$ & .12 & $.41 * *$ & .12 & $.36 * *$ \\
\hline 1976 & $.26 * *$ & $.41 * *$ & .13 & $.62 * *$ \\
\hline
\end{tabular}

\section{5. - Discussion}

La réalisation d'une seconde pousse chez le Pin maritime est le résultat de deux processus consécutifs et indépendants, l'aptitude à la réaliser et l'élongation elle-même : les résultats montrent que le pourcentage d'individus ayant un second cycle d'une famille n'est pas lié à l'élongation secondaire de ces individus.

1) L’aptitude exprimée en fréquence d'expression d'une seconde pousse connaît une forte variation annuelle (de $12 \%$ à $53 \%$ ); dans des conditions plus difficiles, elle garde ses facultés d'expression avec une intensité non négligeable. Malgré une décroissance avec l'âge, elle se conserve jusqu'à un âge relativement élevé pour autant que des greffes d'arbres adultes puissent en témoigner : ce témoignage reste toutefois discret, les verticilles très fins disparaissent très rapidement. Au plan génétique, elle est héritable, stable dans le temps et dans l'espace. 
2) Quand l'élongation secondaire s'exprime, elle représente une proportion importante de l'élongation totale ( 25 p. 100). Ce rapport est conservé à un âge adulte et reste important dans des conditions de sécheresse estivale (19 p. 100). Dans des conditions stationnelles optimales, ce rapport connaît une certaine variation d’année en année (de 16 p. 100 à 30 p. 100). Par contre, en conditions plus difficiles il reste remarquablement stable. Au plan génétique, l'élongation secondaire est héritable mais beaucoup moins stable d'année en année que la fréquence d'expression. La stabilité d'un milieu à l'autre est nulle.

L'expression du polycyclisme joue un rôle important dans l'accroissement total annuel. Elle lui confère un avantage sur l'accroissement total des individus restés monocycliques. Si laptitude se prolonge au cours des années, lavantage est cumulé. A l’opposé, quand l’aptitude ne s'exprime pas l'année suivante, l'avantage est perdu : la perte pouvant être plus importante que le gain réalisé l'année précédente. Par ailleurs l'avantage est augmenté par une amélioration du milieu : de $9 \%$ dans des conditions de dunes à 20 p. 100 dans des conditions de landes semi-humides. Sur ce dernier aspect, les résultats sont assez voisins de ceux de T.D. RuDor.PI (1964) sur Pinus banksiana : l'augmentation de la durée de la photopériode va de pair avec la plus-value obtenue par les polycycliques.

Comment se réalise l'accroissement annuel en hauteur du Pin maritime? L'ensemble des résultats ne permet pas de dégager des mécanismes expliquant l'ensemble des phénomènes impliqués.

- Les figures 3 et 4 montrent clairement que l'élongation annuclle est liée à un type de croissance propre au génotype considéré. On peut parler de croissance de type «polycyclique» et de type «monocyclique».

- A lintérieur même d’un sous-ensemble (polycyclique par exemple) la croissance globale d'une année donnée se décompose en croissance primaire et secondaire antagonistes, complémentaires ou le plus généralement indépendantes (cf. tableau de corrélation 4).

Par ailleurs, d'une manière plus générale, l'apparition d'un second cycle peut affecter indifféremment l'élongation de la pousse primaire de la même année ou de l'annéc suivante.

En face de ces résultats expérimentaux, il y a l'interprétation générale de la croissance des pins. Que ce soient l'accroissement primaire ou secondaire, la pousse dépend surtout de l'initiation des unités élémentaires mais aussi de leur allongement. Pour le Pin maritime, l'initiation des articles de la pousse primaire de l'année $\mathrm{n}$ se réalise à la fin de l'été de l'année $\mathrm{n}-1$, celle des articles de la pousse secondaire à la même époque ou au printemps de l'année $n$.

Quand les conditions environnementales sont telles que les phases du développement végétatif de la tige principale peuvent se réaliser successivement au cours de l'année (élongation primaire, élongation secondaire, initiation des éléments de l'année suivante dans le bourgeon), croissance primaire et croissance secondaire sont indépendantes ou complémentaires. A l'opposé, un arrêt prématuré de la croissance primaire ou une initiation réduite des articles de la pousse primaire de lannée suivante dues à des conditions climatiques particulières expliquerait les corrélations négatives entre les deux types de pousses d'une même année ou de deux années consécutives avec l'hypothèse que les génotypes réagissent différemment à ces conditions. 
Une autre interprétation des corrélations négatives entre croissance primaire et secondaire peut être donnée par les modalités d'expression morphogénétique du second cycle. La liaison négative entre pousse primaire et secondaire d'une même année serait-elle due à l'initiation d'une seconde pousse au cours du printemps de la même année, pendant que l'élongation primaire se réalise? Par contre, la liaison négative entre pousse secondaire de l'année $n$ et pousse primaire de l'année $n+1$ correspondrait-elle à l'initiation du second cycle l'automne précédent la saison de végétation? Celui-ci prédéterminé, s'allongerait quelles que soient les conditions de l'année. L'élongation s'effectuant après l'élongation primaire, plus tardivement dans la saison de végétation repousserait le début de l'initiation de la pousse primaire de l'année suivante : moins de «stem units» sont initiés et l'élongation primaire de l'année suivante est moindre.

Dans le premier cas, l'élongation de la pousse secondaire réduirait l'élongation de la pousse primaire de la même année. Dans le second cas, elle diminuerait le nombre d'articles dans le bourgeon et affecterait indirectement la croissance primaire de l'année suivante.

\section{6. - Conclusion}

Au plan de la sélection, en ne considérant aucun autre caractère que l'accroissement en hauteur, le polycyclisme apparaît comme un critère de sélection largement positif. A la limite, il pourrait justifier une sélection massale indirecte en faveur de la vigueur. Cependant, les liaisons génétiques positives du polycyclisme avec d'autres critères (CRISAN, 1977), comme l'écart à la verticalité, ou la fourchaison condamneraient cette attitude; elle aboutirait à la sélection d'individus de mauvaise forme. Par ailleurs, le polycyclisme occasionne un nombre plus élevé de verticilles et de nouds qui déprécient d'autant la qualité du tronc.

Le caractère labile du polycyclisme, la forte variabilité inter-annuelle de l'expression du polycyclisme d'une part, et la différence de comportement des polycycliques et des monocycliques d'autres part contribuent à augmenter l'instabilité des réponses des génotypes aux variations climatiques annuelles. Sans doute, le polycyclisme joue-t-il un rôle important dans l'interaction génotype $X$ année (KREMER, 1981). Enfin, il crée un bruit de fond non négligeable dans l'estimation des paramètres génétiques (héritabilité). En particulier, l'importance de la variance de dominance $(25 \%$ de la variance génétique) de l'accroissement en hauteur ne serait qu'un artefact du polycyclisme.

L'utilisation du polycyclisme en sélection pose donc un double problème :

- de choix de caractère comme critère de sélection compte tenu de ses corrélations positives avec la croissance et avec la fourchaison et l'écart à la verticalité;

- de méthodologie compte tenu de sa participation supposée à l'interaction génotype $\times$ année et de la surestimation de la variance de dominance. 


\author{
Summary \\ Genetic control of height growth in Pinus pinaster
}

\title{
I. - Influence of polycyclism
}

Results are based on informations coming from several experimental trials : clonal test. maternal progeny test, and biparental progeny test. Observations were made on several years and locations.

Multinodal height growth pattern was separated in two characters :

- frequency of second flush $(F)$;

- second shoot length when expressed (E).

Both characters are heritable, stable in space and time. But stability of frequency is better than stability of length.

Length of the second flush is an important part of total annual height growth : trees with lammas shoot grow better than trees without lammas shoot. Superiority of trees with second flush is increased when lammas shoot is expressed during several years and when environmental conditions are better.

Phenotypic correlations between first and second flush are negative positive or more generally equal to zero. Same values were found for estimations of genelic correlations between first and second flush for the same year.

All these results show that expression of second flush depends on several factors (environmental conditions, morphogenesis of lammas shoot and growth pattern).

Finally estimations of genetic parameters are based by multinodality : heritabilities are underestimated and dominance variance overestimated.

KeY-words : Multinodality, height growth, Pinus pinaster, genetic control, stability.

\section{Références bibliographiques}

Allia R.M., Scarbrougit N.M., 1970. Morphology and length colrelated in terminal flushes of longleaf saplings. U.S.D.A. Forest service research paper, 50-53.

Baradat Ph., 1975. The juvenile mature genetic correlations. Their importance for understanding changes in physiology of tree crops. I.U.F.R.O. physiological genetics conference, Edinburgh.

Cannili, M.G.R., Thompson S., Lines R., 1976. An analysis of inherent differences in shoot growth within some north temperate conifers. In tree physiology and yield improvement, Eds M.G.R. Cannell and F.T. Last, Academic press, p. 173-205.

Castaing J.PH., Illy G., 1966. Rythme saisonnier de croissance en diamètre et en hauteur chez le Pin maritime. Bull. Soc. bot. Fr., 114, 173-179.

David R., 1968. Trois aspects de la physiologie du Pin maritime : la genèse de la racine et de la tige, la formation de l'oléorésine. In Colloque sur la physiologie de larbre, 1966. Mémoire Société Botanique de France, 1968, 137-164.

Debazac E.F., 1963. Morphologie et sexualité chez les Pins. Rev. for. fr., 14, 4, 293-303.

Elston R.C., 1977. Estimating « Heritability» of a dichotomous trait. Biometrics, 33, 1, 231-233. 
HinkelmanN K., Stern K., 1960. Kreuzungspläne zur Selektionzüchtung bei Waldbaümen. Silvae Genet., 9, 121-133.

Kremer A., 1981. Déterminisme de la croissance en hauteur du Pin maritime. 11. Comportement interannuel. Interaction génotype $\times$ année. Ann. Sci. for. (proposé pour publication).

LanNer R., 1970. Origin of the summer shoot of pinyon pines. Can. J. Bot., 48, 1759-1765.

Lanner R., 1976. Patterns of shoot development in Pinus. In Tree physiology and yield improvement, Eds M.G.R. Cannell and F.T. Last, Academic Press, p. 233-243.

LANNer R., 1978. Development of the terminal bud and shoot of slash pine saplings. For. Sci., 24, 2, 167-179.

Mauge J.M., Alazard P., Castaing J.PH., Levadou D., 1976. Critère de sélection pour la croissance en hauteur chez le Pin maritime. Ann. Rech. sylvic., A.F.O.C.E.L., 331-350.

RudolPH T.D., 1964. Lammas growth and prolepsis in jack pine in the lake states. For. science monograph, 6, 1-70.

SEarle S.R., 1971. Linear models. John Wiley and Sons, New York, London, Sydney.

Tallis G.M., 1959. Sampling errors of genetic correlations calculated from the analysis of variance and covariance. Aust. Jour. of Stat., 1, 35-43.

TePper H.B., 1963. Leader growth of young pitch and shortleaf Pines. For. Sci., 9, 344-353. 